\title{
Enabling Biomaterials Translation in Academia: Redefining Value Proposition with Updated Regulations
}

Authors: Didier Letourneur*1, Kieran Joyce*2, ${ }^{3}$, Cédric Chauvierre ${ }^{1}, Y^{*}$ es Bayon ${ }^{4}$, Abhay Pandit ${ }^{2}$.

\section{Affiliations:}

1) Université de Paris, INSERM U1148, LVTS, Université Sorbonne Paris Nord, X Bichat Hospital, 46 rue $\mathrm{H}$ Huchard, F-75018 Paris, France

2) CÚRAM, SFI Research Centre for Medical Devices, Biomedical Sciences Building, National University of Ireland Galway (NUI Galway), Galway, Ireland.

3) School of Medicine, National University of Ireland Galway (NUI Galway), Galway, Ireland.

4) Sofradim Production, A Medtronic Company, Trévoux, France

*These authors contributed equally to this work.

Keywords: Biomaterials, Tissue Engineering, Translation, Medical Device Regulation, Innovation.

\begin{abstract}
Academic institutions are becoming more focused on translating new technologies for clinical applications. A transition from "bench to bedside" is often described to take basic research concepts and methods to develop a therapeutic or diagnostic solution with proven evidence of efficacy at the clinical level while also fulfilling regulatory requirements. The regulatory environment is evolving in Europe with transition and grace periods for the full enforcement of the Medical Device Regulation 2017/745 (MDR), replacing the Medical Device Directive 93/42/EEC (MDD). These new guidelines increase demands for scientific, technical and clinical data with reduced capacity in regulatory bodies creating uncertainty in future product certification. Academic translational activities will be uniquely effected by this new legislation. The barriers and threats to successful translation in academia can be overcome by strong clinical partnerships, close-industrial collaborations, and entrepreneurial programs, enabling continued product development to overcome regulatory hurdles, reassuring their foothold of medical device development.
\end{abstract}




\section{Introduction}

The landscape for academic translation is evolving to align with recent and upcoming changes in regulatory policies. Historically, the efficiency of University technology transfer is generally inferior, characterized by protracted timescales, and low adaptation of Invention disclosure forms (IDFs) towards patents and licenses. Less than half of IDFs will be transformed into patents while only $15 \%$ of original disclosures will be licensed for development and commercialization. ${ }^{[1]}$ This difficulty in transforming innovative ideas from academic research into industry-produced marketable products can be attributed to the high-risk of product success and the uncertainty of the return on investment, such as the cost of patents. ${ }^{[2,3]}$ Industry-based ventures of medical devices accounted for $82 \%$ of all devices obtaining regulatory approval in clinical trials. Collaborative engagement with industry has been shown to incredibly beneficial, as the rate of regulatory approval rate doubles in academic-industrial collaborative ventures. ${ }^{[4]}$ The main hurdle to overcome after regulatory approval is securing financial resources which can be overcome by defining the unmet clinical need and setting out a clear plan to ensure both clinical integration and subsequent reimbursement. ${ }^{[5]}$ The transition into the new regulatory environment in Europe with the full enforcement of the Medical Device Regulation 2017/745 (MDR) will even further challenge the development of emerging technologies into the marketplace.

\section{Where do academic institutions currently stand on the developing landscape of Medtech regulation?}

Academic institutions are gaining a foothold in successful translational endeavors in recent years having a stake in at least a third of all developed devices (Fig 1). ${ }^{[4]}$ Academic research has been the driving force behind the inception of emerging technologies and early development of potential products. ${ }^{[6]}$ Academic centers are focused on implementing multidirectional and multidisciplinary translational research capabilities, with the priority of fulfilling key performance indicators and publication requirements from funding agencies while improving commercialization productivity and bridging translational gaps. ${ }^{[7]}$ Innovation from academic laboratories can be fully realized to have a meaningful impact in the clinic and community through collaborative efforts of discovery and development from both academic and industrial and clinical input. ${ }^{[8]}$ 
Clinicians and industrials share common views for medical device development. These clinical/industrial-oriented projects require research steps to provide innovative solutions or preclinical validations for clinical needs-these factors alone or in combination, drive industrial production towards clinical validation of an end-product. Many companies have graduated from relying on internal $R \& D$ to external collaboration, supported by governmentled initiatives to promote open-innovation projects. ${ }^{[9]}$ Academia-industry joint activities can be characterized as formal (consulting, contract and collaborative research) and informal (academic discourse, guidance and advocacy, and networking with academic and clinical researchers) ${ }^{[10]}$

For instance, Lansac et al. (Vascular Surgery, X. Bichat Hospital, INSERM U1148, Paris) proposed and patented the idea of a new open and suturable aortic ring using previously accredited material components, to reduce the dilated diameter of the aortic root while maintaining expandability. ${ }^{[11]}$ Together with an industrial partner and a research laboratory located within the same hospital, they developed prototypes, performed in vitro tests to evaluate their safety and mechanical effectiveness for a minimum life span of 15 years, and efficacy in sheep up to 6 months after implantation. ${ }^{[11]}$ The clinicians then compared them to the current best clinical practice (mechanical valve replacement), in a prospective multicenter and international randomized prospective study (CAVIAAR Study: Aortic Valve Conservation in Aortic Insufficiency and Root Aneurysms). Positive clinical outcomes on 233 patients (89.9\% survival at seven years) have now led to the 10-year follow-up with more than 5,000 patients (58 centers from 17 countries) undergoing this surgical approach using a medical device in humans (AVIATOR- AorticValve repair InternATiOnal Registry) which is nearing completion.

International and inter-sectoral partnerships are essential for successful clinical translation into a global market. ${ }^{[12]}$ Initiatives such as The European Clinical Research Infrastructure Network (ECRIN), should facilitate multinational clinical research among scientific collaborations and consortia across Europe. ECRIN provides services to support clinical trials and ensure standard operation procedures are consistently implemented, creating reproducible high-quality data while overcoming resource constraints ${ }^{[13]}$ Although industrial partnerships are vital to driving technological advances towards the market, nongovernmental bodies such as ECRIN fulfil an important role to ensure clinical research and innovation are scientifically substantiated, transparent, and meet demanding regulatory stipulations. 
Recent years have seen increasing regulatory and safety requirements in the EU, fierce generic drug competition in the pharmaceutical industry, and the growing complexity of products in the pipeline. Stringent regulations are becoming too cumbersome to fulfill, while the policy is dictating what interventions are adopted. Manufacturing challenges and increasing R\&D costs, meanwhile, are making drug and device development less attractive to multinational companies. This deterrence is reflected by a slowing in industry innovation and a reduction in R\&D funding models. Shortly, we can expect additional challenges with an everchanging landscape, but the focus will remain on innovation potential and differentiation value combined with demonstrable benefit.

\section{EU Medical Device Regulation Impact on Industry and Innovation}

Any medical devices, including biomaterials, intended to be used in the clinics should comply with current regulations for human use. The regulatory environment is evolving in Europe with transition and grace periods for the full enforcement of the Medical Device Regulation 2017/745 (MDR), replacing the Medical Device Directive 93/42/EEC (MDD).

Why is this happening? The change has been motivated to ensure a consistently high level of health and safety protection for European citizens and to adapt to technological and scientific progress. New medical devices will have to comply without exception to new Regulation from May 2021 (postponed due to COVID-19 crisis) and currently approved medical devices under the MDD will have to be approved in conformity with the MDR, by Apr 2024 at the latest, to be placed on the market after this date. The MDR is highly descriptive, with more details and new requirements as illustrated by the number of pages, which jumps from 95 for the former Directives to 175 for the new regulation.

What is not changing? The MDR relies on the same necessary regulatory process, by including these crucial stages of medical device lifecycle: i) product design and manufacturing controls, ii) safety and performance evidence, iii) conformity assessment to in force directives, regulations and harmonized standards involving qualified notified bodies, iv) CE marking and v) vigilance and post-market follow-up.

What is new? The MDR is, in fact, a modernized and strengthened system with significant impacts on the regulation of high-risk devices, notified bodies and clinical evidence generation. Briefly, high-risk devices such as joint replacements, materials in contact with 
spine or central circulatory system are up-classified, i.e. to comply with stricter requirements, which would mean extended time to market access. Notified bodies will instruct any medical device filing for market approval, in a much more rigorous way, especially on clinical evidence. This would, most probably, prolong the time of the review process. A significant change of the MDR is a greater need for clinical investigations according to dedicated European rules and strengthened clinical data transparency.

What is expected by the new MDR in terms of clinical investigation? Clinical data should be more detailed. They must, in principle, be sourced from clinical studies on the device, be supported by published literature or specific and/or strengthened by the relevant post-market experience. The clinical benefit must be demonstrated, either for the patient and/or for clinical practice. Sole demonstration that the device "works" is not enough. Clinical investigations under MDR are mandatory for implants and Class III devices, with limited exceptions. Legacy products found compliant with the MDD before May 2021 may not require additional clinical investigations if they already benefit from sufficient clinical evidence. Clinical evaluation pathways involving equivalency may still be used; however, with less flexibility, while equivalence rules will be presented in the next paragraph. Moreover, postmarket surveillance is a generalized requisite of constant update of clinical evaluation, based on 'active surveillance' of how the device performs in their real clinical life. Patient protection and transparency are strengthened with clinical data, increasingly available to the public, and to competitors, in the new EU database, Eudamed, which is planned to be implemented in 2022. ${ }^{[14]}$

Eudamed will record essential information of medical devices, including certificates and their status, conformity assessment procedures, a summary of safety and clinical performance, vigilance information (e.g. reports of a severe adverse event or device deficiency that occurs during a clinical investigation and post-market surveillance and their associated plans of corrective actions), clinical investigation information (i.e. sponsor, purpose, status, approval, summary).

What do equivalent medical devices mean under MDR? The Revision 4 update to MEDDEV $2.7 / 1^{[15]}$ gave a detailed meaning of equivalence that is consistent with the expectations seen in the MDR. It includes three distinct dimensions: clinical, technical \& biological. Briefly and non-exhaustively, equivalent devices should treat the same disease condition, with the same intended purpose and the same body site and, in the same target 
populations. They should show similar designs and technical features, and importantly, all their components - i.e. materials and/or substances - in contact with biological fluids or human tissues should be the same, and patient-contacting parts may require physicochemical characterization to establish the equivalency. The MDR meaning of equivalence is stricter than the US FDA meaning for 510(k)-regulated devices, relying on the same intended use and similar technological characteristics. ${ }^{[16]}$

What are the main expected impacts of MDR? CE mark dossier submissions should be delayed, particularly for new devices for which equivalency already CE marked devices may no longer represent a possible pathway for the pre-market clinical evaluation. They depend more and more on pre-market clinical data to be generated, not only to show safety but also performance. As mentioned above, this situation differs from US 510(k) approval of new products with in vitro/bench and animal data versus existing predicates to support equivalence in addition to proofs of product safety (e.g. biocompatibility studies according to ISO 10993). Equivalence criteria are distinctly different for incremental product evolution in Europe (CE Mark under MDR) vs the US for devices falling into $510(\mathrm{k})$ products categories. The CE certification time of new products is also expected to increase to check the compliance to all additional requests, such as the 'second review' of the manufacturer's clinical evaluation by the notified bodies. For devices already CE marked under the MDD, they should be all certified under MDR no later than May 2024, and should, notably, systematically comply with the new MDR requirements on clinical evidence generation (see above). Consequently, devices may lose their CE mark approval, with pros and cons for manufacturers, healthcare providers and patients. For example, like cons, devices approved for minimal volume clinical indications with the MDD CE mark may be at risk of disappearing due to the quality and quantity of available clinical data to support post-market surveillance requirements. And more generally, the time of the first certification or certification renewal should also be affected by the slowly increasing number of MDR accredited notified bodies with fourteen to the date, just a few months before the end of the MDD to MDR transition period, in May $2021,^{[14]}$ which may have a substantial impact, at least for the next coming years.

About expected evolution of clinical evidence sources? Clinical evidence sources should most certainly expand, notably to tackle MDR post-market surveillance requirements, as effectively and efficiently as possible. Randomized controlled clinical trials (RCT) and metaanalysis of RCT have undoubtedly a very high value, by allowing the evaluation of a new 
treatment with minimal biases versus carefully selected groups. ${ }^{[17]}$ Generating continuous clinical evidence should provide equivalent additional value on medical devices regarding their safety, efficacy - including the long-term - efficacy and absence of non-desirable effects. This can be achieved by national databases and registries but should be more rewarding if efforts are coordinated and synchronized. ${ }^{[18]}$ This is supported by the International Medical Device Regulators Forum (IMDRF), an international voluntary group of medical device regulators. ${ }^{[18]}$ Real-world data and real-world evidence are further substantiated by the FDA with the $21^{\text {st }}$ Century Cures Act passed in 2016, and issues of guidance to the use of real-world data and real-world evidence to support regulatory decision-making. ${ }^{[19]}$ But it is also important not to lose sight of the expectations of post-market surveillance and to be aware of the limits of the different approaches to generating clinical data, i.e. the value that they can actually produce. For example, registries for abdominal hernia surgeries have been developed following pan-national initiatives (e.g. Herniamed in German-speaking countries, Germany, Austria \& Alemannic Switzerland) and clinical societies (e.g. EuraHS in Europe, AHSQC in the USA). Herniamed was successful at accumulating enough surgery cases with more than seven commercial meshes used for intraperitoneal on-lay mesh (IPOM) hernia repair to clearly show that one of them led to higher hernia recurrence rate, one year after surgery. ${ }^{[20]}$ Potentially, other tools can be used, nationwide clinical data sharing database initiatives (e.g. German Medical Informatics in Germany and a Health data hub in France) with enough depth of individual patient information to support retrospective and continuous clinical studies. ${ }^{[21]}$ Recommendations by national and European agencies as well as by clinical societies are expected to guide post-market surveillance and the notion of what defines an acceptable, yet achievable, level of clinical evidence.

\section{How Academia Should Move to be a Stronger Translation Initiator \& Stakeholder - a SWOT analysis considering update MDR}

A SWOT analysis identifies the internal strengths and weaknesses of university-based translation, as well as its external opportunities and threats (Fig. 2).

\section{Strengths}


Universities are in a unique position to partake in translational research with their intrinsic link to the clinic through institutional undergraduate and postgraduate programs integrating clinical students in bench and biomedical research. The pool of MD/PhD and broadly based PhD investigators capable of performing this translational research is gradually being increased with new integrated MD-PhD programs becoming accessible. ${ }^{[22,23]}$ Furthermore, patient involvement and considerations into recommendations relevant to researchers' activities are valuable to gain experiential knowledge. ${ }^{[24]}$ Close industry collaborations are valuable to mitigate risk and ensure a focused approach towards project selection and fast track translation. ${ }^{[2]}$ There must be a clear discourse from the outset between an academic institution and a company from the beginning to agree on the timely release of data and a roadmap towards patenting. ${ }^{[25]}$

This symbiotic relationship between academia and industry better aligns medical devices with market expectations and commercial success, when an affordable and reimbursable product is needed. Therefore, optimization of production costs and the early discussions with relevant authorities on reimbursement strategies play a crucial role in transforming these products into the standard of care therapeutics.

Specific niches in R\&D processes have been identified in which these academicindustrial partnerships are most likely to see the best return on investment for all stakeholders. These research units are best equipped to deliver on small-scale product development in the early adoption phase of new technology. Academic institutions are becoming more involved in developing combination devices of an industry product with new delivery vehicles or through the expansion of existing, university generated platforms of materials, molecules and disease targets. Such an example is Cambridge-based Healx, which utilizes artificial intelligence to enhance drug discovery in rare diseases. ${ }^{[26]}$ In the academicindustry partnerships, the focus shifted towards composite device(s) for renewal of patents to ward off competition from generic formulations. ${ }^{[27]}$ Most new medical product "innovations" introduced to the market are not innovations, but incremental changes to existing product classes, demonstrating improved efficacy and fewer off-target effects. The MDR includes articles addressing devices that contain a biologically active component on the market. These drug-device combinations are separated into two categories: 1) Devices incorporating a substance or drug that, if used stand-alone, would be considered a medicinal product and the action of the substance in the device is principal (Article 1.8) and 2) Devices 
that administer a medicinal product, in which the combination device forms a single integrated non-reusable product (Article 1.9). Any implantable substrate will be classified as a medical device or as an Advanced Therapy Medicinal Product (ATMP) according to its working principles and/or components and will be regulated accordingly.

Industries expand into new fields by collaborating with academic labs with prior expertise in such fields. ${ }^{[28]}$ Lipocoat, a spin-out from the University of Twente, has patented a phospholipid-coating for implants for drug-delivery and to improve osteointegration, securing $€ 3$ million in funding to date. ${ }^{[29]}$

Universities with a strong portfolio of university-industry linkages to reflect the scope of activities and the types of expertise better aligned with their institutional goals and funding criteria. A balanced portfolio of projects in risks and cost is crucial to bridge the gap to sustaining batch manufacturing for preclinical and first-in-human trials. To ensure success, these activities require exceptional support for protocol development and regulatory submissions. Regulatory bodies must also be involved early in these ventures, as they will specify the use of specific models for pharmacological and toxicological studies. ${ }^{[30]}$

\section{Weaknesses}

Translation in academia has traditionally been slow with few healthcare practices recommended by research institutes actually being implemented, and it frequently takes more than fifteen years for evidence-based research recommendations to be translated into standard practice in healthcare institutions, in an environment that has a high turnover in student and post-doctoral workers based on the nature and structure of funding calls and position durations. ${ }^{[1]}$

The barriers to translation are interconnected, where the inadequate design of research programs means easy adoption into a healthcare setting is not facilitated. Furthermore, poor or inadequate marketing to stakeholders may mean failure to recognize an evident value proposition for the new idea. The act of translation is one of the core problems in itself, as apparent from the disconnect of translating the evidence-based results from scientifically literate researcher to a common language understood by the clinicallyoriented practitioner and stakeholders in the form of tangible results and potential clinical outcomes. ${ }^{[31]}$ Conversely, while scientists have difficulty expressing the importance of their 
concepts in real-world terms, clinicians also fail to translate clinical needs to researchers in a comprehensible way. ${ }^{[32]}$

Increasing regulatory demands are resource-intensive. For example, methodical iteration and statistical validation of molecules, targets, pathways, doses, formulations/prototypes, and series of preclinical testing with certified quality-controlled methods are often difficult to find in classic academic laboratories. ${ }^{[33]}$ The additional inspection, including increased onus on clinical evaluation, instructions for use, and postmarket surveillance will make some potential value propositions unsustainable. This clinical evaluation must be carried out conforming to the requirements stipulated in Chapter VI. Altogether, these increased restrictions and stipulations will increase the cost of translating devices to market, highlighting the need for increased investment from the industrial or staterun enterprise.

\section{Opportunities}

Industry collaborations and formal entrepreneurial training activities equip academic entrepreneurs with the relevant knowledge and experience to understand regulatory challenges associated with clinical translation. ${ }^{[34]}$ These skills allow them to augment their awareness of potential opportunities while further appreciating the resource configurations development pathways required to pursue newly recognized opportunities. Universities have been developing new educational programs and framework to support innovation with Bedside-to-Bench-and-Back programs, spurred by the mounting evidence that a disconnect between basic science and clinical researchers hinders entrepreneurial ventures. ${ }^{[35]}$ In the US, project-oriented curricula have emerged within academic institutions to promote entrepreneurship; such is the case with SPARK-Stanford, where more than half of the incubated projects are licensed every year, to be implemented in the clinic or further commercialized. ${ }^{[36]}$ An equivalent innovation program, Bioinnovate, has been installed in NUI Galway and seen similar success in its fellowship program. EMBO medical, specializing in single-shot reliable embolization devices, was Bioinnovate's first spin-out company in which was acquired ClearStream Technologies for almost €43.5 million in 2016. Similarly, EU programs, educational curricula and dedicated summer schools have been developed. The European Institute of Innovation \& Technology (EIT) Health has been successful in facilitating 
collaborations between academics, clinicians and industry, resulting in 122 start-ups that have secured $€ 27.9 \mathrm{M}$ in capital investment. ${ }^{[37]}$

In Ireland, for example, the Enterprise Ireland Commercialisation Fund Programme supports researchers in academic institutions that aim to convert the outputs of state-funded research into innovative licensed products, services and start-up companies. A researcher can apply for feasibility grants, commercialization funds and clinical innovation awards. Academic institutions have a unique opportunity to provide a hub for parties to create multi-disciplinary teams from medicine, science, engineering, business and law faculties with mutually aligned goals. ${ }^{[38]}$ Educating researchers with a commercialization mindset can have a significant impact on how researchers view and act on translational opportunities. ${ }^{[38]}$

Devices approved for minimal volume clinical indications with the MDD CE mark may be at risk of disappearing due to the quality and quantity of available clinical data to support post-market surveillance requirements. And more generally, the time of the first certification or certification renewal should also be affected by the slowly increasing number of MDR accredited notified bodies with fourteen to the date, just a few months before the end of the MDD to MDR transition period, in May 2021, ${ }^{[14]}$ which may have a substantial impact, at least for the next coming years. Academic-led projects can address the need for increased, highquality clinical data by elucidating the mechanism of action, strengthening the safety and efficacy of a device through increased confirmatory preclinical investigations. ${ }^{\text {[39] [40] }}$

Academic research can play a valuable role in influencing health policy by developing guidelines with network-driven national initiatives that include clinicians, scientists, industry experts, investors, regulatory experts and policymakers to guarantee clinical adoption, costeffectiveness and return on investment. The scope of translation can be broadened to include social impact and increased public engagement through film-making, classroom workshops, public events and media coverage. These outputs inform the community to enact change in societal values and policy to advocate health and well-being. A scientifically informed population will promote awareness and understanding for continued funding from national agencies. ${ }^{[41]}$ This communication between researchers and end-users creates a complex model of translational research, as an iterative, multidirectional and dynamic process, increasing the likelihood of technology adoption by the target group. ${ }^{[7]}$ Community-driven research strategies focused on interdisciplinary collaborations, aim to more efficiently integrate proven health services interventions into clinical practice. ${ }^{[42]}$ 


\section{Threats}

Concerns are already being raised regarding the time-to-market in Europe for new medical devices. While the EU moves to replace the MDD with the MDR, the US FDA have released new strategies aiming to "reduce the time and cost of generating clinical evidence, typically the most expensive and lengthy regulatory requirement for marketplace entry." This move comes at a time when few MDR accredited notified bodies have been approved for processing the expected increase in re-applications to conform to additional requirements. These factors combined could make EU market access difficult and costly, compelling start-ups to translate into other markets.

The new Articles of the MDR describe standards that must be conformed to, representing a significant increase in onus and responsibility on academics and all stakeholders. Additionally, a designated regulatory compliance officer must be appointed within an organization, with demonstrable prior experience and expertise in medical device regulation and compliance. While the classification system remains standard, definitions have narrowed and been altered for some products, which will cause some devices to be reclassified to higher classes (under Classification Rule 21 and Annex I). Furthermore, previously unclassified or exempt devices will now be classified and regulated within the scope of the new legislation. This will incur high cost on existing products licensed by universities, potentially leading to discontinuation if capital investment in such projects has not foreseen these increased demands for regulatory approval.

The EU MDR will impose the obligation to produce clinical data for the CE marking of biomaterial-based implants, as they are high risk devices. Compared to the MDD, The MDR puts significantly more focus on clinical evaluation and post-market surveillance. This new legislation eliminates the principle of equivalence for market approval, requiring more sophisticated trials and increased costs for clinical evaluation. To improve translation in academia, dedicated teams are necessary to screen the commercialization potential of projects with a health economic assessment to decide on further progression towards translation. ${ }^{[43]}$ Across Europe, there is limited consensus clinical and economic analysis in health-technology assessment guidelines. ${ }^{[4]}$ The evidence-based identification of unmet clinical needs and market research engages investors with an attractive value proposition to 
improve the efficiency of the translational system to move progress outside the academic sphere through start-ups formed of a multidisciplinary team. ${ }^{[45]}$ Early input from stakeholders is crucial in guiding the development of biomaterials to market. To this end, industrial partners, funding bodies (private and/or public), clinicians and end-users should be consulted early, defining clear milestones with go/no-go decisions, to protect the integrity of the product for more successful translation.

\section{Conclusion}

Academic institutions remain a significant contributor to the creation and translation of clinicready and patient-focused medical devices while developing the next generation of industryrelevant, publicly engaged researchers to become an anchor for applicable industry research. While regulatory systems are evolving with uncertainty looming over the certification and market access, translational activities remain attractive ventures. Universities are wellpositioned to attract sufficient investment to meet increased regulatory demands through fruitful industrial partnerships and dedicated state-run funding schemes. The barriers and threats to successful translation in academia can be overcome by strong clinical partnerships, close-industrial collaborations, dedicated funding schemes and entrepreneurial programs, enabling continued product development to overcome regulatory hurdles, reassuring their foothold of medical device development. 


\section{Acknowledgements}

The authors would like to take this opportunity to thank Michael Gelinsky, Prof. Peter Dubruel for organizing the plenary session with Yves Bayon at the Annual Conference of the European Society for Biomaterials (ESB), 2019, in Dresden, from which this review has emanated. Works performed by DL and CC are supported by INSERM, University of Paris and University Sorbonne Paris Nord. DL has shares in the Siltiss Company for the development of Tissue Engineering products. Works performed by KJ are supported by CMNHS Scholarship, NUI Galway and the European Regional Development Fund and Science Foundation Ireland under Ireland's European Structural and Investment Fund, Grant Number 13/RC/2073.

\subsection{References}

[1] Z. S. Morris, S. wooding, J. Grant, J. R. Soc. Med. 2011, 104, 510.

[2] M. Abou-El-Enein, G. N. Duda, E. A. Gruskin, D. W. Grainger, Trends Biotechnol. 2017, 35, 100.

[3] G. N. Duda, D. W. Grainger, M. L. Frisk, L. Bruckner-Tuderman, A. Carr, U. Dirnagl, K. M. Einhäupl, S. Gottschalk, E. Gruskin, C. Huber, et al., Sci. Transl. Med. 2014, 6, $264 \mathrm{~cm} 12$.

[4] H. J. Marcus, C. J. Payne, A. Hughes-Hallett, A. P. Marcus, G. Z. Yang, A. Darzi, D. Nandi, BMJ 2016, 353, i2587.

[5] J. F. Farragher, M. J. Elliott, S. A. Silver, Z. Lichner, A. Tsampalieros, Can. J. Kidney Heal. Dis. 2015, 2.

[6] J. M. Merigó, C. A. Cancino, F. Coronado, D. Urbano, Scientometrics 2016, 108, 559.

[7] A. L. van der Laan, M. Boenink, Heal. Care Anal. 2015, 23, 32.

[8] R. J. Kleiman, M. D. Ehlers, Mol. Biol. Cell 2019, 30, 2741.

[9] J. West, A. Salter, W. Vanhaverbeke, H. Chesbrough, Res. Policy 2014, 43, 805.

[10] M. Perkmann, V. Tartari, M. McKelvey, E. Autio, A. Broström, P. D’Este, R. Fini, A. Geuna, R. Grimaldi, A. Hughes, et al., Res. Policy 2013, 42, 423.

[11] E. Lansac, I. Di Centa, F. Raoux, N. Bulman-Fleming, A. Ranga, A. Abed, M. Ba, A. Paolitto, D. Letourneur, A. Meddahi-Pellé, J. Thorac. Cardiovasc. Surg. 2009, 138, 718.

[12] A. De Pieri, S. Ribeiro, D. Tsiapalis, D. Eglin, M. Bohner, P. Dubruel, P. Procter, D. I. Zeugolis, Y. Bayon, J. Mater. Sci. Mater. Med. 2018, 29, 129.

[13] C. Ohmann, S. Canham, J. Demotes, G. Chêne, J. Lauritsen, H. Martins, R. V. Mendes, 
E. B. Nicolis, A. Svobodnik, F. Torres, Contemp. Clin. Trials Commun. 2017, 5, 153.

[14] European Commision, "European database on medical devices (EUDAMED) | Internal Market, Industry, Entrepreneurship and SMEs," can be found under

https://ec.europa.eu/growth/sectors/medical-devices/new-

regulations/eudamed_en, 2020.

[15] European Commision, “MEDDEV 2.7/1 revision 4, Clinical evaluation: a guide for manufacturers and notified bodies," can be found under https://ec.europa.eu/docsroom/documents/17522/attachments/1/translations/en/r enditions/native, 2016.

[16] G. A. Van Norman, JACC Basic to Transl. Sci. 2016, 1, 277.

[17] R. Collins, L. Bowman, M. Landray, R. Peto, N. Engl. J. Med. 2020, 382, 674.

[18] HQIP, "Proposal for a Medical Devices Registry - HQIP," can be found under https://www.hqip.org.uk/resource/proposal-for-a-medical-devices-registry/\#.XrcTdNKjcs, 2020.

[19] Food and Drug Administration, "Real-World Evidence / FDA," can be found under https://www.fda.gov/science-research/science-and-research-special-topics/realworld-evidence, 2020.

[20] F. Köckerling, T. Simon, M. Hukauf, A. Hellinger, R. Fortelny, W. Reinpold, R. Bittner, Ann. Surg. 2018, 268, 1097.

[21] M. Cuggia, S. Combes, Yearb. Med. Inform. 2019, 28, 195.

[22] L. F. Brass, M. H. Akabas, JCl Insight 2019, 4, e133009.

[23] L. F. Brass, M. H. Akabas, L. D. Burnley, D. M. Engman, C. A. Wiley, O. S. Andersen, Acad. Med. 2010, 85, 692.

[24] M. Boenink, L. van der Scheer, E. Garcia, S. van der Burg, Nanoethics 2018, 12, 181.

[25] V. Drozdoff, D. Fairbairn, Cold Spring Harb. Perspect. Med. 2015, 5, a021014.

[26] B. Bhhatarai, W. P. Walters, C. E. C. A. Hop, G. Lanza, S. Ekins, Nat. Mater. 2019, 18, 410.

[27] H. Ahn, Second Generation Patents in Pharmaceutical Innovation., Nomos Verlagsgesellschaft, 2014.

[28] M. R. Barnes, L. Harland, S. M. Foord, M. D. Hall, I. Dix, S. Thomas, B. I. WilliamsJones, C. R. Brouwer, Nat. Rev. Drug Discov. 2009, 8, 701.

[29] B. J. van Meer, H. de Vries, K. S. A. Firth, J. van Weerd, L. G. J. Tertoolen, H. B. J. 
Karperien, P. Jonkheijm, C. Denning, A. P. IJzerman, C. L. Mummery, Biochem. Biophys. Res. Commun. 2017, 1, 6.

[30] C. S. Pridgeon, C. Schlott, M. W. Wong, M. B. Heringa, T. Heckel, J. Leedale, L. Launay, V. Gryshkova, S. Przyborski, R. N. Bearon, et al., Arch. Toxicol. 2018, 92, 557.

[31] J. H. Linehan, A. Chaney, in Sci. Transl. Med., American Association For The Advancement Of Science, 2010, pp. 63mr6-63mr6.

[32] L. L. Restifo, G. R. Phelan, Dis. Model. Mech. 2011, 4.

[33] C. Chauvierre, R. Aid-Launais, J. Aerts, F. Chaubet, M. Maire, L. Chollet, L. Rolland, R. Bonafé, S. Rossi, S. Bussi, et al., Mar. Drugs 2019, 17, 699.

[34] D. W. Grainger, H. C. van der Mei, P. C. Jutte, J. J. A. M. van den Dungen, M. J. Schultz, B. F. A. M. van der Laan, S. A. J. Zaat, H. J. Busscher, Biomaterials 2013, 34, 9237.

[35] P. D. Loftus, C. T. Elder, T. D’Ambrosio, J. T. Langell, Clin. Transl. Med. 2015, 4, 15.

[36] S. Gehr, C. C. Garner, Cell 2016, 165, 765.

[37] S. Ribeiro, M. Ricci, A. Von Der Lieth, Y. Bayon, D. I. Zeugolis, S. Pelayo, I. Marque, L. Pazart, in Heal. 2019 - 12th Int. Conf. Heal. Informatics, Proceedings; Part 12th Int. Jt. Conf. Biomed. Eng. Syst. Technol. BIOSTEC 2019, 2019.

[38] S. A. Maher, R. Kyle, B. F. Morrey, M. J. Yaszemski, in J. Am. Acad. Orthop. Surg., 2019.

[39] J. Kimmelman, J. S. Mogil, U. Dirnagl, PLoS Biol. 2014, 12, e1001863.

[40] R. E. Meyer, P. F. Griner, J. Weissman, Proc. Assoc. Am. Physicians 1998, 110, 513.

[41] Science Foundation Ireland, "SFI discover | Engagement | Science Foundation Ireland," can be found under https://www.sfi.ie/engagement/sfi-discover/, 2020.

[42] T. A. Battaglia, K. M. Freund, J. S. Haas, N. Casanova, S. Bak, H. Cabral, R. A. Freedman, K. B. White, S. C. Lemon, Contemp. Clin. Trials 2020, 93, 106007.

[43] M. Abou-El-Enein, G. Bauer, P. Reinke, M. Renner, C. K. Schneider, Trends Mol. Med. 2014, 20, 632 .

[44] M. Blüher, S. J. Saunders, V. Mittard, R. Torrejon Torres, J. A. Davis, R. Saunders, Front. Med. 2019, 6, 278

[45] H. D. Volk, M. M. Stevens, D. J. Mooney, D. W. Grainger, G. N. Duda, Sci. Transl. Med. 2015, 7. 


\section{Academic translation}

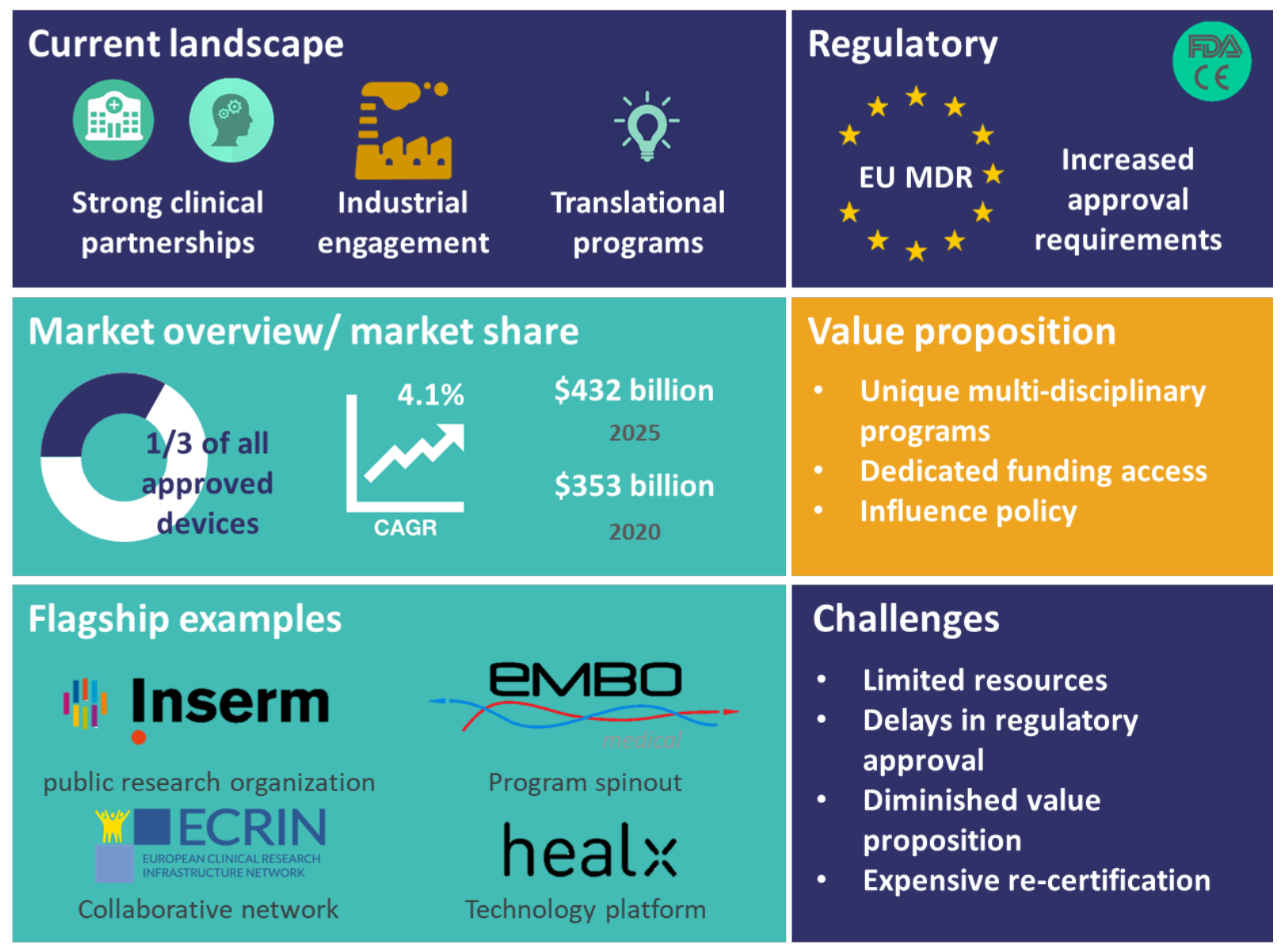

Figure 1. Insight into the current landscape for academic translation. Academia focuses on activities centered on clinical collaborations, industrial partnerships and translational programs. In 2016, universities were involved in the development of one-third of all devices approved by regulatory bodies. This market is expected to grow with a compound annual growth rate (CAGR) of $4.1 \%$ over the next five years. Examples of key translational activities are embodied by public research organizations, university-based spinout companies, collaborative networks and technology platforms. The unique value proposition of universities remains attractive despite growing challenges in device approval and path to market. 


\section{STRENGTHS}

- Clinical partnerships

- Combination devices

- Basic science platforms

- Strong industrial linkages

\section{OPPORTUNITIES}

- Translational programs to

promote entrepreneurship

- Dedicated funding schemes

- Clinical validation

- Community-driven strategies

\section{WEAKNESSES}

- Generating value proposition

- Finite resources to meet regulatory requirements

- Underfunded commercialisation

\section{THREATS}

- Increased time to market

- Competitive US approval

- Increased approval costs reduce value proposition

- Re-certification demands

Figure 2. SWOT analysis of University-based translational activities. The perceived weaknesses and threats to university-based translation are offset by the perceived strengths and opportunities in light of the developing landscape of regulatory legislation. 